\title{
Neuroticism and extraversion mediate the relationship between having a sibling with developmental disabilities and anxiety and depression symptoms
}

\author{
Dr Lindsay E Murray (Corresponding Author) \\ Department of Psychology \\ University of Chester \\ Parkgate Road \\ Chester, CH1 4BJ UK
}

Tel: (0044) 1244513479

Fax: (0044) 1244511323

Email: 1.murray@chester.ac.uk

Dr Linda O’Neill

Department of Psychology

University of Chester

Parkgate Road

Chester, CH1 4BJ UK

Tel: (0044) 1244513479

Fax: (0044) 1244511323

Email: 1.oneill@chester.ac.uk

Declaration of interests: none

Contributors:

LM and LON designed the study, LON collected the data, LM and LON analysed the data, wrote and approved the paper.

This research did not receive any specific grant from funding agencies in the public, commercial, or not-for-profit sectors. 


\section{Abstract \\ Background}

Children growing up with a sibling with disabilities report higher levels of depression and anxiety symptoms as adults. Here, we examined whether personality characteristics also play a part in mediating this relationship.

\section{Method}

We tested for differences in personality traits between 132 individuals who have a sibling with developmental disabilities and 132 closely matched comparisons.

Results

Differences in Big Five factors of personality were demonstrated across the disability groups and between the disability groups and the comparisons, especially in Extraversion, Neuroticism and Openness. Individuals growing up alongside a sibling with developmental disabilities have a higher tendency to experience anxiety and depression symptoms, and this research is the first demonstration that personality traits mediate this relationship. Specifically, Neuroticism is a strong mediator of anxiety while both Neuroticism and Extraversion contribute mediating effects toward the development of depression.

\section{Limitations}

Our study made use of self-report methodology which, although having recognized limitations, is more reliable than parental reports. Given the cross-sectional nature of our design, we were not able to examine pre-existing developmental factors that may have influenced the participant's propensity to particular personality traits and affective 
disorders. However, we obtained a large sample and closely matched participants to examine differences between those with a sibling with disabilities and those without.

\section{Conclusions}

As such, differences in personality traits have important implications for the understanding and treatment of siblings presenting with anxiety and depression symptoms. We recommend that intervention should target those high in Neuroticism among individuals who have a sibling with disabilities, and that more social support is put in place for siblings to mitigate their tendency towards introversion and buffer them against psychological maladjustment.

Keywords: personality, sibling, developmental disabilities, anxiety, depression, mediation 
Introduction

Affective disorders likely develop due to a complex interaction between genetic, environmental and developmental factors (Hartley and Casey, 2013; Rutter and Silberg, 2002); consequently, any adverse circumstances can have more or less of an effect on an individual's life largely depending on the perception of the situation by the individual (McAdams and Pals, 2006; McCrae et al., 2000). In childhood, the family context is paramount and, although siblings of individuals with developmental disabilities usually have unproblematic education and employment prospects, they can later experience challenges in maintaining romantic relationships (Wolfe et al., 2014). We have shown that children growing up with a sibling with disabilities report higher levels of depression and anxiety symptoms as adults (Redacted for blind review, 2016a).

In this study, we examine whether personality characteristics also play a part in the experiences of siblings of individuals with developmental disabilities. Indicators of the Big Five dimensional traits of personality appear early in life and across different cultures, before social indicators such as language (Slobodskaya et al., 2012). Personality traits are arguably endogenously-based, showing high levels of stability and predictability over the lifespan (McCrae, 2002; McCrae et al., 2000), or they manifest mean level changes over time (Gow et al., 2005). Such lifetime plasticity of personality traits could be attributed to broader life experiences (Edmonds et al., 2008; Srivastava et al.,2003) such as family constellation and, to an extent, age and sex differences (Gow et al., 2005). Even to the extent that traits remain reasonably stable, their expression will be contextually dependent (Clark et al., 2003). 
Different explanations for the associations between personality traits and affective disorders have been suggested (Watson et al., 2005): either that temperament affects affective disorders, or conversely that the development of the affective disorders affects the personality traits, or that personality and affective disorders share the same underlying developmental causes, so that neither is responsible for the onset of the other. Individuals can also be genetically predisposed to affective disorder symptomatology (McCrae et al., 2000).

Neuroticism is a significant predictor of affective disorders with high scorers predisposed towards negativity ranging from unhappiness and lowered life satisfaction to clinical anxiety and/or depression (Hutchinson and Williams, 2007; Jylhä and Isometsä, 2006; Kotov et al., 2010; Lönnqvist et al., 2009; Scott et al., 1995; Trull and McCrae, 2005; Widiger and Anderson, 2003). Lönnqvist et al. (2009) suggest that Neuroticism can predict affective disorders once the personality of an individual has stabilized, usually following young adulthood. Importantly, those high in Neuroticism are more likely to suffer from affective disorders when faced with stressful life events. Neuroticism may act as an underlying vulnerability factor in the development of depressive symptoms (Lang and Farmer, 2007) and can become particularly provoked when daily hassles operate as a mediating factor (Hutchinson and Williams, 2007), even if an increase in such hassles is a perception rather than a reality. 
In contrast to Neuroticism, high levels of Extraversion are associated with positive emotionality and sociability (McCrae et al., 2000; Watson et al., 2005) and lower levels of anxiety and depression, together with a decreased use of psychiatric services (Jylhä and Isometsä, 2006). The American National Co-morbidity Survey (Kessler et al., 1996) found Extraversion had significant, modest, negative associations with anxiety and depression; Neuroticism was highly positively associated with anxiety and depression, while a third member of the Big Five - Openness - had no associations with affective disorders.

High levels of childhood adversity significantly increase the risk of correspondingly high levels of Neuroticism and negative affect (Rosenman and Rodgers, 2006) with girls more likely to score higher on Neuroticism and on anxiety and depression measures, in contrast to boys who score higher on Extraversion (Del Barrio et al., 1997). Siblings of individuals with developmental disabilities arguably experience additional stressors in their everyday family lives (Mascha and Boucher, 2006), and they are less likely to engage in extra-curricular activities (Barak-Levy et al., 2010), potentially leading to relationship and behavioral problems (e.g., Giallo and Gavidia-Payne, 2006; Hastings, 2007; Hastings and Petalas, 2014). However, there are also positive consequences, including greater empathy (Cuskelly and Gunn, 2003; Meadan et al., 2010), and positive relationships with their disabled sibling (Hodapp et al., 2010). Less sibling rivalry and conflict is also reported (Kaminsky and Dewey, 2002) among siblings of children with Autism Spectrum Disorder (ASD) and, indeed, these siblings can broaden their own experience by teaching their siblings new skills, learning calming techniques to use with 
their siblings, and raising awareness in others through their understanding and appreciation of their siblings (Angell et al., 2012).

In terms of Agreeableness and Conscientiousness, siblings of those with Down's Syndrome (DS) score higher than a comparison group, perhaps due to socialisation processes requiring them to act as caregivers to their disabled siblings at an early age and leading to greater feelings of family solidarity (Cuskelly and Gunn, 2003). In contrast, although not sibling studies, Austin (2005) found increased levels of Neuroticism and lower levels of Extraversion and Agreeableness in a study of individuals with the broader autism phenotype (BAP). Similarly, increased levels of Neuroticism and lowered levels of Extraversion and Conscientiousness have also been found in a BAP group

(Wakabayashi et al., 2006). These findings are relevant to our study as the BAP relates to family members who may share some subclinical characteristics of ASD even if not diagnosed. Other research, however, has found no differences in the personality characteristics of sibling and comparison groups (Lounds-Taylor et al., 2008).

\section{Rationale of the present study}

To our knowledge there has been no empirical research carried out to ascertain if mediation occurs between personality traits, having a sibling with disabilities, and anxiety and depression symptomatology. It is reasonable to suppose that high levels of stress arising from the acute or chronic health and behavioral problems of their siblings, or increased responsibility in the home including caretaking of their disabled siblings when children (Dyke et al., 2009; Meyer, 1997; Moore et al., 2002; Powell and Gallagher, 
1993; Randall and Parker, 1999) - could lead to increased levels of Neuroticism (Rosenman and Rodgers, 2006). Similarly, the siblings might have had fewer opportunities for social activities (Barak-Levy, et al., 2010; Seltzer et al., 2009), perhaps resulting in lowered perceptions of social support, particularly for those with BAP (Jobe and Williams-White, 2007), potentially leading to lower levels of Extraversion.

Alternatively, siblings who may be extraverted but in need of support may just be unable to find the time to engage in more social activities.

In addition, witnessing negative behaviors in their sibling with disabilities while understanding that the sibling has little control over their behavior, and also witnessing outsiders' reactions (Seltzer et al., 2009) might increase levels of Openness in the sibling as they learned to tolerate different perspectives and the conduct or reactions of others. They might also experience higher levels of Conscientiousness as they learned to respond to their sibling's medical or behavioral routines and consequently learned high levels of personal control (Angell et al, 2012).

We have previously demonstrated that siblings of individuals with developmental disabilities had significantly greater vulnerability towards higher anxiety and depression symptomatology and that this could vary depending upon the type of disability their sibling has (Redacted for blind review, 2016a). We recognise that simply having a sibling with disabilities is not sufficient reason per se to experience anxiety or depression. Likewise, it would be an over-simplification to suppose that being a sibling of an individual with a disability is predictive of a particular pattern of personality 
characteristics. Therefore, we carried out tests that examined for main effects and mediation analyses in order to ascertain whether personality traits play a role in the vulnerabilities towards affective disorders of siblings of individuals with disabilities (henceforth referred to as the sibling group). Consequently, the above literature lead to the following research questions being investigated:

Firstly, we hypothesized that individual sibling groups (DS, ASD, Prader-Willi Syndrome (PWS), Disabled with Unknown Aetiology (DUA)) would differ from the comparison group on the five personality traits as follows: the individual sibling groups will report higher levels of Neuroticism (Moore et al., 2002; Powell and Gallagher, 1993; Rosenman and Rodgers, 2006) and Openness (Seltzer et al., 2009) and lower levels of Extraversion (Stoneman et al., 1988) than the comparison group. This is predicted due to potentially higher levels of stress or hassles (Hutchinson and Williams, 2007) relating to the disability that the sibling group encountered when younger and carried forward into adulthood. We also predicted that the traits of Agreeableness and Conscientiousness would be higher in the individual sibling groups than the comparison group, due to the caregiving towards their sibling with disabilities that they possibly experienced in the early years (Cuskelly and Gunn, 2006; Shiner and Caspi, 2003).

Secondly, we hypothesized that Neuroticism would mediate the relationship between being in the sibling versus the comparison group and anxiety and/or depression symptomatology. Neuroticism is the trait most closely associated with anxiety and depression (Hutchinson and Williams, 2007; Trull and McCrae, 2005; Watson et al., 
2005) and could help to explain the relationship between having a sibling with disabilities and increased anxiety and/or depression symptomatology. Thirdly, we hypothesized that Extraversion would mediate the relationship between being in the sibling versus the comparison group and anxiety and/or depression symptomatology. Previous research highlighted low levels of Extraversion to be a robust and consistent marker of depressed affect and interpersonal anxiety (Watson et al., 2005), although not as influential as Neuroticism (Jylhä and Isometsä, 2006). Fourthly, we wanted to take a more exploratory approach with the remaining three personality traits as the literature does not clearly link Openness, Agreeableness or Conscientiousness with anxiety and depression or with having a sibling with disabilities (e.g. Kessler et al., 1996; McCrae and Costa, 1991; Ready and Robinson, 2008; Watson et al., 2005). As an example, in one sense, the sibling group might be expected to have higher Openness, having experienced, and had to deal with, unusual life events regarding their sibling with disabilities. On the other hand, their exposure to sometimes negative behaviors on the part of their sibling, and the reactions of others to these, can lead to embarrassment or resentment (Angell et al, 2012), which could have the opposite effect on levels of Openness. We therefore decided to put forward for mediation analysis only those traits which specifically correlated with having a sibling with disabilities.

\section{Method}

\section{Participants}

As detailed in (Redacted for blind review, 2016 a, b), our sample included 132 participants who were siblings of individuals with developmental disabilities, comprising 
specifically siblings of those with Down's Syndrome (DS; $n=59$ ), Autism Spectrum Disorder (ASD; $n=31$ ), Prader-Willi Syndrome (PWS; $n=26$ ) or Disabled with Unknown Aetiology (DUA; $n=16$ ). Recruitment was via various UK disability charities, which included Down's Syndrome Association, the National Autistic Society, the PraderWilli society and SIBS, who issued invitations to potential participants to contact us for recruitment in the study. The age range varied from 19 to 71 years (mean $34.46 \pm 11.04$ ). The sex split was 34 (25.8\%) male and 98 (74.2\%) female. A further 132 participants were included as a comparison group on the basis of their case-by-case matching to the sibling group on sex and age; and thereafter on marital status, socio-economic status, employment status and educational attainment. The comparison group was contacted through the university, local businesses, friends and family. The age range for this group was $18-71$ with a mean age of $37.61 \pm 12.58$. The sex split was $34(25.8 \%)$ male and 98 (74.2\%) female. The criterion for inclusion in this group was that they should have at least one sibling but no sibling with disabilities.

\section{Measures}

Participants were asked for demographic information including sex, age, marital status, highest education level and details of their sibling with disabilities, if this applied to them, and for their responses to the following measures relating to affective symptoms and personality:

The Hospital Anxiety and Depression Scale (HADS, Zigmond and Snaith, 1983) was used to assess affective symptoms. We found high reliability for both anxiety (Cronbach's alpha reliabilities were .84 for the SDI group and .86 for the comparison 
group) and for depression (.70 and .80 respectively) using this 14-item self-report instrument. The scale had been previously validated using participants from a nonpsychiatric background (Snaith, 2003) and the cut-off points of the scale are useful indicators for borderline or clinical anxiety and depression.

The International Personality Item Pool (Goldberg, 1999; IPIP, 2001) is a 50-point scale, available in the public-domain (Goldberg et al., 2006), and was used to assess participants' position on the Big 5 traits of Extraversion (E), Agreeableness (A), Conscientiousness (C), Neuroticism (N) and Openness to Experience (O). The IPIP has proven reliability and validity (e.g. Ypofanti et al., 2015; Zheng et al., 2008). Typical questions to establish the participants' strength on the trait were "Am the life of the party" (E), "Feel little concern for others" (A), “Am always prepared" (C), "Get stressed out easily"(N) and "Have a rich vocabulary"(O). The participants responded using a 5point Likert scale ranging from 'very inaccurate' to 'very accurate'. The respective Cronbach alpha statistics for the Big 5 traits were $.88, .81, .81, .89$ and .80 for the SDI group and $.73, .79, .78, .87$ and .79 for the comparison group.

\section{Design and Procedure}

The data gathered were part of a larger research project examining anxiety and depression symptoms in the adult siblings of those with disabilities population. Therefore, the participant recruitment procedures, data collection method and findings regarding anxiety and depression are described in detail elsewhere (Redacted for blind review, 2016a). The study obtained approval from the departmental ethics committee and all participants 
provided written informed consent. This was a cross-sectional, between-subjects study design, completed by self-report. We used the disability type as the grouping factor for multivariate analysis with five levels: DS, ASD, PWS, DUA and the comparison group. Univariate analysis tested for significant differences in personality traits between the individual sibling groups and the comparison group using one-way ANOVAs, with Eta Squared $\left(\eta^{2}\right)$ to examine effect sizes. Due to insufficient sample size per disability group, we combined the sibling groups into one overall sibling group and conducted correlation analysis between anxiety and depression scores, personality scores and having a sibling with disabilities. For reasons of parsimony and to increase statistical power (Cohen, 1988; Spinhoven et al., 2011), we put forward for mediation analysis only those traits which specifically correlated with having a sibling with disabilities. Using the PROCESS macro tool for SPSS (Hayes, 2012), we utilized bootstrapping methods in order to estimate bias-correct asymmetric confidence intervals (CI) of the indirect effects. We used 5000 randomised resampling with replacement, and regarded the mediation as statistically significant if the CI did not include zero (Preacher and Hayes, 2008). When analysing anxiety, we controlled for the demographic variable of sex, which we have previously identified as an influencing factor (Redacted for blind review, 2016a) and similarly the variables of sex and age were controlled for when analysing depression. The alpha level for all analyses was set at $p \leq .05$.

\section{Results}

\section{Differences in personality traits between the disability groups}


Table 1 shows the demographic and personality scores according to disability subgroup. In terms of demographic information, all of the groups had more female respondents than male. Tukey's post hoc analyses showed that those whose siblings had DUA were older than the other groups whose siblings have a disability; specifically, older than those whose sibling had DS (mean difference $=9.28, \mathrm{SE}=3.23, p=.04$ ), ASD (mean difference $=10.31, \mathrm{SE}=3.53, p=.03$ ) and PWS (mean difference $=11.85, \mathrm{SE}=3.65, p$ $=.01$ ). Similarly, those individuals who remain undiagnosed were older than those who have DS (mean difference $=8.36, \mathrm{SE}=2.80, p=.02), \mathrm{ASD}($ mean difference $=11.27, \mathrm{SE}$ $=3.07, p=.02$ ) and PWS (mean difference $=12.01, \mathrm{SE}=3.15, p=.01$ ). 
Note: SDI=Sibling of Disabled Individual; Severity of disability - rated either mild, moderate, severe or profound

Table 1: Demographics, anxiety, depression and personality scores by sibling disability subgroup

\begin{tabular}{|c|c|c|c|c|c|c|c|}
\hline$\overline{\text { Siblings }}$ & $\begin{array}{l}\text { DS group } \\
(N=59)\end{array}$ & $\begin{array}{l}\text { ASD group } \\
(N=31)\end{array}$ & $\begin{array}{l}\text { PWS group } \\
(N=26)\end{array}$ & $\begin{array}{l}\text { DUA } \\
(N=16)\end{array}$ & $\begin{array}{l}\text { Comparison } \\
(N=132)\end{array}$ & Test statistic & $\operatorname{Eta} \mathrm{Sq}\left(\eta^{2}\right)$ \\
\hline & Mean (SD) & Mean (SD) & Mean (SD) & Mean (SD) & Mean (SD) & & \\
\hline Anxiety & $8.12(4.54)$ & $10.23(4.45)$ & $10.23(4.14)$ & $9.50(2.97)$ & $6.94(4.03)$ & $F(4,258)=6.24, p<.001$ & 0.09 \\
\hline Depression & $3.85(2.80)$ & $4.81(2.94)$ & $4.73(3.34)$ & $5.75(3.36)$ & $3.19(3.06)$ & $F(4,259)=4.44, p=.01$ & 0.06 \\
\hline Extraversion & $3.24(0.87)$ & $3.20(0.75)$ & $3.43(0.72)$ & $2.91(0.80)$ & $3.49(0.67)$ & $F(4,246)=3.67, p=.006$ & 0.06 \\
\hline Agreeableness & $4.01(0.62)$ & $4.23(0.50)$ & $4.30(0.57)$ & $3.78(0.62)$ & $4.09(0.59)$ & $F(4,247)=2.93, p=.02$ & 0.05 \\
\hline Conscientiousness & $3.84(0.65)$ & $3.56(0.83)$ & $3.65(0.60)$ & $3.46(0.56)$ & $3.60(0.61)$ & $F(4,240)=1.60, p=.18$ & 0.02 \\
\hline Neuroticism & $3.05(0.93)$ & $3.34(0.68)$ & $3.17(0.82)$ & $3.38(0.53)$ & $2.92(0.73)$ & $F(4,255)=3.05, p=.02$ & 0.05 \\
\hline Openness to Experience & $3.74(0.59)$ & $3.91(0.63)$ & $3.59(0.51)$ & $3.39(0.48)$ & $3.49(0.59)$ & $F(4,247)=3.21, p=.01$ & 0.05 \\
\hline Sex (\% female) & 66.1 & 90.3 & 69.2 & 81.3 & 74.2 & ns & \\
\hline Age of SDI & $33.22(9.01)$ & $32.19(11.52)$ & $30.65(9.70)$ & $42.50(14.59)$ & $37.03(12.34)$ & $F(4,254)=4.42, p=.002$ & 0.06 \\
\hline Age of sib with disability & $32.57(6.36)$ & $29.66(12.48)$ & $28.92(11.02)$ & $40.98(13.20)$ & - & $F(3,127)=5.76, p=.001$ & 0.12 \\
\hline Severity of disability & $2.47(0.71)$ & $2.57(1.55)$ & $2.65(0.63)$ & $2.69(0.79)$ & - & ns & \\
\hline SDI older than sib \% & 55.9 & 58.1 & 53.8 & 31.3 & - & ns & \\
\hline
\end{tabular}


Initial examination of personality scores looked for differences between the individual disability sibling groups and the comparison group. MANOVA indicated significant overall group effects for the personality factors (Wilks $k=0.84, F(20,714)=1.89, p=$ $.01)$. According to Cohen (1988), a medium effect size for Eta Squared is 0.059 and so most of the personality variables, with the exception of Conscientiousness, are in this range. Therefore, we can see that $6 \%$ of the change in reporting of Extraversion levels can be accounted for through disability of sibling type. Similarly 5\% of Agreeableness, Neuroticism and Openness can also be accounted for by disability type (see Table 1). Univariate analyses, using Tukey's post hoc analysis, showed that those with siblings with DUA reported significantly lower levels of Extraversion than the comparison group (mean difference $=0.64, \mathrm{SE}=0.21, p=.02$ ), while siblings of those with PWS reported significantly higher levels of Agreeableness than those siblings with DUA (mean difference $=0.52, \mathrm{SE}=0.18, p=.04)$. Those whose siblings have ASD reported significantly higher levels of Neuroticism and Openness than the comparison group (mean difference $=0.44, \mathrm{SE}=0.16, p=.05 ;$ mean difference $=0.36, \mathrm{SE}=0.12, p=.03$, respectively) and also higher levels of Openness than the DUA group (mean difference $=$ $0.52, \mathrm{SE}=0.19, p=.04)$. All other pairwise comparisons were not significant $(p>0.05)$

\section{Correlational analysis}

Anxiety and depression symptoms were co-morbid and each had strong associations with having a sibling with disabilities. Anxiety, depression and having a sibling with disabilities were all associated with each other. Having a sibling with disabilities was 
also associated with the personality variables of Extraversion, Neuroticism and Openness (see Table 2). 
Table 2: Correlations between the significant principal variables under study $(N=264)$

\begin{tabular}{|c|c|c|c|c|c|c|c|c|c|c|}
\hline & & 1 & 2 & 3 & 4 & 5 & 6 & 7 & 8 & 9 \\
\hline 1. & Group & -- & & & & & & & & \\
\hline 2. & Anxiety & $.25^{* *}$ & -- & & & & & & & \\
\hline 3. & Depression & $.21 * *$ & $.57 * *$ & -- & & & & & & \\
\hline 4. & Extraversion & $-.20 * *$ & $-.21 * *$ & $-.37 * *$ & -- & & & & & \\
\hline 5. & Agreeableness & -.04 & -.08 & $-.22 * *$ & $.38 * *$ & -- & & & & \\
\hline 6. & Conscientiousness & .02 & -.05 & -.03 & -.08 & $.22 * *$ & -- & & & \\
\hline 7. & Neuroticism & $.18^{*}$ & $.70 * *$ & $.47 * *$ & $-.28 * *$ & $-.17 * *$ & -.02 & -- & & \\
\hline 8. & Openness & $.12 *$ & .09 & -.11 & $.21 * *$ & $.20 * *$ & $.19 * *$ & .12 & -- & \\
\hline 9. & Sex & -.02 & $.20 * *$ & $.15^{*}$ & .00 & $.27 * *$ & .03 & $.25^{* *}$ & -.11 & -- \\
\hline 10. & Age & $-.14 *$ & -.04 & $.13^{*}$ & -.06 & .02 & $.17 * *$ & -.09 & -.04 & .06 \\
\hline
\end{tabular}

Key: * Correlation is significant at the 0.05 level; ** significant at the 0.01 level; Group = participants having a sibling with disabilities coded 1 , comparison group coded 0

\section{Mediation analyses}

We conducted Multiple Parallel Mediation modelling to analyse the simultaneous contribution of personality traits as mediators in the relationship between having a sibling with disabilities and both anxiety and depression symptomatology. Bootstrapping analyses, using Hayes' (2012) PROCESS macro tool, were conducted to examine for potential mediating effects of the personality variables of Extraversion, Neuroticism and Openness. As shown in Figure 1, we found a significant indirect effect of having a sibling with disabilities on anxiety symptoms through Neuroticism, ab $=0.92$, BCa CI [0.26, 1.62], which accounted for nearly $40 \%$ of the total effect, $\mathrm{PM}=.39$. Extraversion $(\mathrm{ab}=-$ 0.03, BCa CI [-0.23, 0.12], PM = -0.01) and Openness $(\mathrm{ab}=-0.01, \mathrm{BCa}$ CI $[-0.12,0.08]$, PM = -0.01) had negligible effects on anxiety. Therefore, having a sibling with 
disabilities predicts higher Neuroticism which, in turn, predicts higher levels of anxiety symptoms.

(Figure 1 here)

Fig. 1. Personality traits as mediators between having a sibling with disabilities and anxiety symptoms.

Note: Path $\mathrm{c}=$ having a sibling with disabilities predicts anxiety. Path $\mathrm{c}^{\prime}=$ having a sibling with disabilities predicts anxiety controlling for personality traits. Path a1-3 = having a sibling with disabilities predicts personality trait. Path b1-3 = personality trait predicts anxiety while controlling for having a sibling with disabilities. Analysis controls for sex. Unstandardized regression weights. Significance level: $*<.05$, $* *<.01, * * *<.001 . \bullet 95 \%$ confidence interval does not include 0 .

Figure 2 also displays a significant indirect effect of having a sibling with disabilities on depression through both Neuroticism, $\mathrm{ab}=0.40, \mathrm{BCa} \mathrm{CI}[0.11,0.77]$, which accounted for roughly a quarter of the total effect, $\mathrm{PM}=.26$ and Extraversion, $\mathrm{ab}=0.21, \mathrm{BCa} \mathrm{CI}$ $[0.06,0.50]$, accounting for $14 \%$ of the total effect $(\mathrm{PM}=0.14)$. Openness, $\mathrm{ab}=-0.09$, BCa CI $[-0.28,0.01]$, accounted for 6\% (PM $=-0.06)$ of the effect on anxiety but, as the CI contained zero, it cannot be regarded as a significant mediator. Therefore, having a sibling with disabilities predicts higher Neuroticism and lower Extraversion which, in turn, predict higher levels of depression symptoms.

(Figure 2 here)

Fig. 2. Personality traits as mediators between having a sibling with disabilities and depression.

Note: Path $\mathrm{c}=$ having a sibling with disabilities predicts depression. Path $\mathrm{c}^{\prime}=$ having a sibling with disabilities predicts depression controlling for personality traits. Path a1-3 = having a sibling with disabilities predicts personality trait. Path b1-3 = personality trait predicts depression while controlling for having a sibling with disabilities. Analysis controls for sex and age. Significance level: $*<.05, * *<.01$, $* * *<.001 . \bullet 95 \%$ confidence interval does not include 0 . 


\section{Discussion}

To our knowledge, these findings are the first to demonstrate that personality traits mediate the relationship between having a sibling with disabilities and anxiety and depression symptoms. Providing partial support for our first hypothesis, we found significant differences in personality scores between the individual sibling groups and the comparison group. As a whole, being in the sibling group was positively associated with Neuroticism and Openness and negatively associated with Extraversion. Those whose siblings have ASD reported significantly higher levels of Neuroticism and Openness than the comparison group, while those whose siblings have DUA reported significantly lower levels of Extraversion than the comparison group. We also found significant differences between the disability groups: those whose siblings had PWS showed higher levels of Agreeableness than those whose siblings had DUA and, similarly, those whose siblings had ASD showed higher levels of Openness than those whose siblings had DUA. There were no significant differences in Conscientiousness between the groups. When the next three hypotheses were examined, we found that Neuroticism was highly significant in mediating the relationship between having a sibling with disabilities and anxiety and depression symptoms. In addition, Extraversion had a more modest, but still significant, influence in mediating depression symptomatology. Openness was not found to play a mediating role.

Our findings for Neuroticism and Extraversion therefore go against Lounds-Taylor et al.'s (2008) study which found no difference for any of the five traits between adult sibling and comparison groups, although the nature of the disabilities differed between studies. Our results broadly support the findings of Austin (2005) and Wakabayashi et al. 
(2006) who both found elevated levels of Neuroticism and lowered levels of Extraversion in their research on those with BAP, although these were not sibling studies. Our findings of higher Neuroticism and Openness (with the exception of the DUA group) scores and lower levels of Extraversion in the sibling group might be explained through Rossiter and Sharpe's (2001) fundamental suggestion that issues surrounding one member of a family - in this case a disabled member - can affect all family members. Siblings of children with disabilities have an increased propensity to experience emotional difficulties and peer problems (Giallo and Gavidia-Payne, 2006), which relate to the trait of Neuroticism, and adverse events that happened in childhood can have an enduring effect of increasing negative affect throughout the lifetime (Rosenman and Rodgers, 2006). These and other researchers therefore stress the necessity for clinical service-providers to be aware of the background of every individual, in order to ensure their long-term welfare.

We found that higher levels of Neuroticism partially mediated the relationship between having a sibling with disabilities and adult anxiety symptomatology; whereas higher levels of Neuroticism and lower levels of Extraversion partially mediated this relationship for depression symptoms. Following on from Clark and Watson's (1991) classic tripartite model identifying the links between negative emotionality and both anxiety and depression, Watson et al. (2005) suggest that Neuroticism, while a consistent marker of psychopathology, is most strongly associated with chronic and persistent symptoms of subjective distress and dysphoria; whereas lowered levels of Extraversion are a more 
consistent marker of depressed affect and social anxiety. Our findings broadly support this.

The sibling group's lower levels of Extraversion could perhaps be due to limited availability of social opportunities when younger because of the potentially problematic behavior of their sibling with disabilities (Barak-Levy, et al., 2010; Seltzer et al., 2009). The taxonomic description of Extraversion involves the extent to which the individual actively seeks out social encounters or tries to avoid them (Shiner and Caspi, 2003). The sibling cohort may have been more likely to be socially withdrawn, due to embarrassment or a protective feeling towards their sibling with disabilities. This could be differentiated from being shy and such social withdrawal could occur, particularly if they had previously been snubbed by others (Muris et al., 2009; Shiner and Caspi, 2003). This has been identified in children whose siblings have ASD, with reports of feelings of loneliness and isolation, especially in males (Lamport and Zlomke, 2014), and it is therefore important that siblings can access support groups to help them overcome these feelings.

We found Openness to be higher in the sibling group compared to comparisons. A description of Openness includes depth of thought and complexity of life events alongside a willingness to try new experiences (Shiner and Caspi, 2003). Adult siblings have been found to be less embarrassed about the situation regarding their sibling with disabilities, less concerned about social stigma and less worried about being isolated from their peers than a comparison adolescent group (Wilson et al., 1992). This implies a 
greater direction towards Openness as the sibling aged; with a willingness to embrace new experiences and not be tied to conforming to social norms (Seltzer et al., 2009). Its potential role as a mediator is worthy of future research: if individuals with a sibling with disabilities can be encouraged to have higher Openness to experiences, this could, in turn, have a protective effect against them developing depressive symptoms.

We found no significant links between having a sibling with disabilities and Conscientiousness or Agreeableness. This was somewhat surprising as it was expected that the sibling group might be significantly higher in Conscientiousness than comparisons due to being used to the more rigid routines required by their sibling with disabilities. Similarly, we expected that they may score more highly on Agreeableness due to their increased experience of caregiving and support (Barak-Levy et al, 2010) (despite the fact that only three participants identified themselves as primary caregivers, they would arguably contribute more than siblings to typically developing individuals). However, Agreeableness is a problematic and contentious factor. Many of the items upon which participants self-report focus on aspects such as trust which are quite different to the qualities of the word 'agreeable' that most people would associate with it. People 'with trust issues' will often have experienced something earlier in life that predisposes them to being less trusting; however, those same people can be very caring and trusting of those closest to them. It is not hard to envisage how the reactions of others to their sibling with disabilities might influence the sibling to be less trusting of others due to their previous experiences. Whilst this is speculative, it may be an area worthy of future research. 
In terms of how the mediation by personality traits might operate, a number of models attempt to explain the aetiological roots of trait-affective disorder relationships (De Bolle et al., 2016; Shiner and Caspi, 2003; Watson et al., 2005). According to the predisposition or vulnerability model, particular personality traits, such as Neuroticism, may increase the probability of developing a clinical disorder. Our identification of Neuroticism as a mediator would seem to fit such a model quite well, with those experiencing their lives with a sibling with disabilities being more likely to exhibit higher Neuroticism which in turn can lead to higher levels of affective symptomatology. Alternatively, a trait such as Neuroticism might exist alongside a disorder, each with its own developmental onset, but may influence the direction or severity of the disorder; such a pathoplasty or exacerbation model could be examined more readily through moderation analysis. In contrast, according to spectrum and continuity models, which form the basis of more integrated frameworks for examining the covariation of stability and consistency of personality and psychopathology, personality and particular affective disorders might share an underlying aetiological basis, possibly genetic, which is influential in the development of both personality and affective disorders. This may particularly apply to those whose sibling has ASD; as siblings manifesting BAP traits are of particular interest due to their shared genes and environmental experience (Howlin et al., 2015; Pisula and Ziegart-Sadowska, 2015). While our data are not longitudinal and cannot directly contribute evidence supporting one of these models over another, we decided to utilize new advances in mediation analyses to provide a detailed test of the role of personality traits as parallel mediators to help to explain the relationship between 
having a sibling with disabilities and increased anxiety and /or depression symptomatology. We do however suggest that longitudinal research into this area would be highly pertinent, and that BAP symptoms be included as a variable of interest. Such longitudinal follow-ups of our sample may permit the testing of the alternative scar model whereby the individuals' existing anxiety or depression symptomatology could change their personality traits, either in the short- or longer-term, and perhaps dependent on their specific experiences of life with their sibling, which therefore warrants more detailed investigation of a qualitative nature.

The likelihood that personality, or at the more basic level, temperament, affects an individual's adaptation to the environment is great (e.g., Rothbart and Bates, 2006). Vulnerability to daily hassles has also been highlighted as important in the development of depressive symptoms in individuals high in Neuroticism (Hutchinson and Williams, 2007), and the mediating influence between Neuroticism and depression symptomatology exerted by daily hassles could be due to such individuals reporting more incidents than those lower in Neuroticism; the authors could not, however, establish if the siblings did actually experience more stressors or just perceived that they did. This line of thinking could help to explain our results; the sibling group might have experienced various stressors including poorer family functioning, lower levels of pro-social behavior (Giallo and Gavidia-Payne, 2006), the need to excel (Moore et al., 2002; Powell and Gallagher, 1993), and increased caregiving tasks, in respect of their sibling with disabilities (Dyke et al., 2009), all of which might have exacerbated the tendencies towards increased Neuroticism levels and development of a vulnerability towards affective 
symptomatology. This, in turn, could escalate to the pathoplasty model (Watson et al., 2005), influencing the severity of the affective disorder; depending upon how many of the aetiological stressors were interacting.

In a similar vein to our study, Spinhoven et al.'s (2011) research tried to assess if Neuroticism and Extraversion predicted anxiety and depression and if life events could then moderate the symptomatology of these conditions. They also found that the impact of Neuroticism was significant in the prediction of depression; whereas high levels of Extraversion had an ameliorating effect on both anxiety and depression symptomatology. However, our study also found that Neuroticism was highly significant in explaining the mediating path between having a sibling with disabilities and anxiety symptoms. Such a difference in findings might be explained by the fact that, while our sibling participants likely experienced many positive aspects, having a sibling with disabilities is a lifelong 'event', an entrenchment effect worthy of further research. This helps to emphasise the different aetiology between the developmental courses of anxiety and depression and that, while there might often be a significant degree of co-morbidity between the disorders, they should usually be examined as separate entities.

Another important aspect of life with a sibling with disabilities is the effect on the parents. Macks and Reeve (2007) found that parental availability for their other children, both emotional and physical, was compromised; a concerning consequence which is realized by the parents themselves (Oruche et al., 2012). Mothers of children with disabilities are also more likely to experience maternal depression than those with 
typically functioning children (Baker et al., 2011; Motamedi et al., 2007) and this would be an interesting factor to investigate further. Rosenman and Rodgers (2006) found significant associations between remembered childhood adversity and high levels of Neuroticism, and that the effects of maternal depression had lasting and fundamental consequences that did not subside with age. The transgenerational transmission effect between maternal depression and negative affect in her offspring is well-established (e.g., Katz et al., 2013; Klucznoik et al., 2016) and this effect might be further exacerbated by the additional situations that the siblings already encounter, thus potentially helping to explain their higher levels of anxiety and depression symptoms. The birth order of siblings would be an interesting aspect to examine in future research; specifically, whether there are differences in personality traits dependent upon whether the sibling was born before or after the sibling with disabilities, and how this relates to their experience and their affective symptomatology.

That Openness tends to be higher in the sibling group - and may in future be identified as a potential mediator in the relationship between having a sibling with disabilities and depressive symptoms - provides some useful future direction for this field. Openness measures facets of personality such as curiosity, imagination, intellect and the willingness to try new experiences (Shiner and Caspi, 2003). The reasons behind the higher scoring on openness by the siblings can be understood due to them possibly having to employ different, quite imaginative, strategies in dealing with potential behaviors in their siblings with disabilities. Also, the sibling group might have learned to become more accepting of the behavior of others that is not similar to their own (Seltzer et al., 2009); particularly 
if these behaviors are met with subliminal or overt disapproval from observers. The lowered levels of Openness shown in the siblings of those individuals who have not been diagnosed may be related to the unknown future that their sibling has, and so, instead of being open and embracing new challenges, it might feel safer for them to close down and protect what they already have. We recommend that such increased levels of Openness could be something to encourage in modifying depressive symptoms in the sibling group, and are therefore useful to be aware of in a clinical setting.

Although our study made use of self-report methodology, which has its limitations, particularly given the sensitive nature of the topics in question, sibling self-report data has been found to be more reliable than parental reports (Guite et al., 2004; Macks and Reeve, 2007). Given the cross-sectional nature of our design, we were not able to examine pre-existing developmental factors that may have influenced the participants' propensity to particular personality traits and affective disorders. While we asked siblings for confirmed medical diagnoses, we did not verify the disability diagnoses ourselves, which could be considered a possible limitation. However, we obtained a large sample and were able to closely match participants to examine differences between those with a sibling with disabilities and those without which is a strength. Although our sample was large, we did not have sufficiently large numbers to represent each disability group and so we combined the different disability types into one overall sibling group for most of the analyses. This limitation should be borne in mind when applying the findings to specific disability groups. Having uncovered interesting mediation roles for the personality traits, we recommend that future research should consider alternative models which take into 
account the inter-relations of other relevant variables and the roles they may play as mediators and/or moderators in the complex links between growing up with a sibling with disabilities and anxiety and depression symptoms.

\section{Conclusions}

Our study provides much needed specificity on how Neuroticism particularly plays an important role in the development of anxiety and depression symptoms. Importantly, having a sibling with disabilities does not mean that individuals are more likely to develop anxiety and depression symptoms per se. Our sibling group reported higher Neuroticism and Openness, together with lower Extraversion than comparisons (Clark et al., 2003; Watson et al., 2005); and two of these personality traits play important mediating roles in the link between having a sibling with disabilities and having greater anxiety and depression symptoms.

Our findings lend further support to the suggestion that personality characteristics be taken into account in clinical settings when patients present with affective disorders (Jylhä and Isometsä, 2006; Widiger and Anderson, 2003). Personality should also form an important focus of investigation for agencies offering support to families with children with disabilities. There is an unmet need for greater sibling support. Where more support is available, particularly from family and close friends, siblings are less likely to succumb to psychological maladjustment (Barrera et al., 2004; Lovell and Wetherell, 2016). Sibling support programmes and groups need to be more widespread to permit more 
sharing of sibling experiences - itself an important direction for future research - and service providers need to find ways to encourage more social interaction, particularly with other siblings in similar situations. Perhaps through recreational opportunities, children living with a sibling with disabilities may be able to form supportive social networks and friendships to mitigate their tendency toward Neuroticism and introversion, and, in turn, anxiety and depression symptoms in later life.

Acknowledgements

We thank all the participants and the charities which aided our recruitment.

Declaration of Conflicting Interests

The authors declared no potential conflicts of interest with respect to the research, authorship, and/or publication of this article.

\section{Funding}

This research received no specific grant from any funding agency in the public, commercial, or not-for-profit sectors.

References 
Angell, M., Meaden, H., Stoner, J. 2012. Experiences of siblings of individuals with Autism Spectrum Disorders. Autism Research and Treatment, Article ID 949586, http://dx.doi.org/10.1155/2012/949586

Austin, E. 2005. Personality correlates of the broader autism phenotype as assessed by the Autism Spectrum Quotient (AQ). Pers Indiv Differ 38, 451-460. DOI: 10.1016/j.paid.2004.04.022

Baker, J., Seltzer, M., Greenberg, J. 2011. Longitudinal effects of adaptability on behavior problems and maternal depression in families of adolescents with autism. J Fam Psychol 25, 601-609. http://dx.doi.org/10.1037/a0024409

Barak-Levy, Y., Goldstein, E., Weinstock M 2010. Adjustment characteristics of healthy siblings of children with autism. J Fam Stud 16, 155-164. DOI: 10.5172/jfs.16.2.155

Barrera, M., Fleming, C., Khan, F. 2004. The role of emotional social support in the psychological adjustment of siblings of children with cancer. Child Care Hlth Dev $30,103-111$.

Clark, L., Vittengl, J., Kraft, D., Jarrett, R. 2003. Separate personality traits from states to predict depression. J Pers Disord 17, 152-172. DOI:

10.1521/pedi.17.2.152.23990

Clark, L., Watson, D. 1991. Tripartite model of anxiety and depression: psychometric evidence and taxonomic implications. J Abnorm Psychol 100, 316-36.

Cohen, J. 1988. Statistical power analysis for the behavioral sciences. New York, NY: Routledge Academic. 
Cuskelly, M., Gunn, P. 2006. Adjustment of children who have a sibling with Down syndrome: Perspectives of mothers, fathers and children. J Intell Disabil Res 50, 917-925. http://dx.doi.org/10.1111/j.13652788.2006.00922.x

De Bolle, M., De Clercq, B., De Caluwe, E. Verbeke, L. 2016. Exploring the complexity of the childhood trait-psychopathology association: Continuity, pathoplasty, and complication effects. Child Y Psy 28, 139-148. DOI: $10.1017 /$ S0954579415000346

Del Barrio, V., Moreno-Rosset, C., Lopez-Martinez, R., Olmedo, M. 1997. Anxiety, depression and personality structure. Pers Indiv Differ 23, 327-335.

Dyke, P., Mulroy, S., Leonard, H. 2009. Siblings of children with disabilities: Challenges and opportunities. Acta Pædiatr 98, 23-24.

Edmonds, G.W., Jackson, J.J., Fayard, J.V., Roberts, B.W. 2008. Is character fate, or is there hope to change my personality yet? Soc Pers Psychol Compass 2/1, 399, 413.

Giallo, R., Gavidia-Payne, S. 2006. Child, parent and family factors as predictors of adjustment for siblings with a disability. J Intell Disabil Res 50, 937-948. http://dx.doi.org/10.1111/j.1365-2788.2006.00928.x

Goldberg, L. 1999. A broad-bandwidth, public domain, personality inventory measuring the lower-level facets of several five-factor models. In I. Mervielde, I. Deary, F. De Fruyt, F. Ostendorf (Eds.), Personality Psychology in Europe Vol. 7 (pp. 7-28). Tilburg, The Netherlands: Tilburg University Press.

Goldberg, L. R., Johnson, J. A., Eber, H. W., Hogan, R., Ashton, M. C., Cloninger, C. R. Gough, H. G. 2006. The International Personality Item Pool and the future of public 
domain personality measures. J Res Pers 40, 84-96.

DOI:10.1016/j.jrp.2005.08.007

Gow, A.J., Whiteman, M.C., Pattie, A., Deary, I.J. 2005. Goldberg's ‘IPIP’ Big-Five Factor markers: Internal consistency and concurrent validation in Scotland. Pers Indiv Differ 39, 317-329.

Hartley, C., Casey, B. 2013. Risk for anxiety and implications for treatment: developmental, environmental, and genetic factors governing fear regulation. Ann NY Acad Sci 1304, 1-13. DOI: 10.1111/nyas.12287.

Hastings, R. 2003. Behavioral adjustment of siblings of children with autism engaged in applied behavior analysis early intervention programs: The moderating role of social support. J Autism Dev Disord 33, 141-150. DOI: 0162-3257/03/0200 0099/0.

Hastings, R., Petalas, M. 2014. Self-reported behavior problems and sibling relationship quality by siblings of children with autism spectrum disorder. Child Care Hlth Dev 40, 833-9. DOI: 10.1111/cch.12131.

Hayes, A. 2012. PROCESS: A versatile computational tool for observed variable mediation, moderation, and conditional process modeling [White paper]. Retrieved from http://www.afhayes.com/public/process2012.pdf

Hodapp, R., Urbano, R., Burke, M. 2010. Adult female and male siblings of persons with disabilities: Findings From a national survey. Intellect Dev Disab 48, 52 62. 
Howlin, P., Moss, P., Savage, S., Bolton, P., Rutter, M. 2015. Outcomes in adult life among siblings of individuals with autism. . J Autism Dev Disord 45, 707-18. DOI: $10.1007 / \mathrm{s} 10803-014-2224-5$.

Hutchinson, J., Williams, P. 2007. Neuroticism, daily hassles, and depressive symptoms: An examination of moderating and mediating effects. Pers Indiv Differ 42, 1367-1378.

International Personality Item Pool 2001. A scientific collaboratory for the development of advanced measures of personality traits and other individual differences. Retrieved July 6, 2005, from http://ipip.ori.org/newBigFive5broadKey.htm.

Jobe, L., Williams-White, S. 2007. Loneliness, social relationships, and a broader autism phenotype in college students. Pers Indiv Differ 42, 1479-1489.

Jylhä, P., Isometsä, E. 2006. The relationship of neuroticism and extraversion to symptoms of anxiety and depression in the general population. Depress Anxiety 23, 281-289.

Kaminsky, L., Dewey, D. 2002. Psychosocial adjustment in siblings of children with autism. J Child Psychol Psyc 43, 225-32.

Katz, S., Hammen, C., Brennan, P. 2013. Maternal depression and the intergenerational transmission of relational impairment. J Fam Psychol 27, 86-95.

Kessler, R.C., Nelson, C.B., McGonagle, K.A., Liu, J., Swarz, M., Blazer, D.G. 1996. Comorbidity of DSM-III-R major depressive disorder in the general population: Results from the US National Comorbidity Survey. Brit J Psychol $168,17-30$.

Klucznoik, D., Attar, C.H., Fydrich, T., Fuehrer, D., Jaite, C., Domes, G... Bermpohl, F. 
2016. Transgenerational effects of maternal depression on affect recognition in children. J Affect Disorders 189, 233-239.

Kotov, R., Gamez, W., Schmidt, F., Watson, D. 2010. Linking "big" personality traits to anxiety, depressive, and substance use disorders: a meta-analysis. Psychol Bull 136, 768-821. DOI: $10.1037 / \mathrm{a} 0020327$

Lamport, D., Zlomke, K. 2014. The Broader Autism Phenotype, social interaction anxiety, and loneliness: Implications for social functioning. Curr Psychol 33, 246-255. DOI 10.1007/s12144-014-9210-0

Lang, K., Farmer, A. 2007. The causes of depression. In: Stein G, Wilkinson G (Eds) Seminars in general adult psychiatry London: The Cromwell Press, pp. 4870.

Lönnqvist, J.E.,Verkasalo, M., Mäkinen, S., Henriksson, M. 2009. High neuroticism at age 20 predicts history of mental disorders and low self-esteem at age 35 . J Clin Psychol 65, 781-790.

Lounds-Taylor, J., Greenberg, J.S., Seltzer, M.M., Floyd, F. 2008. Siblings of adults with mild intellectual deficits or mental illness: Differential life course outcomes. J Fam Psychol 22, 905-914. http://dx.doi.org/10.1037/a0012603

Lovell, B., Wetherell, M. 2016. The psychophysiological impact of childhood autism spectrum disorder on siblings. Res Dev Disabil 49-50, 226-234.

McAdams, D., Pals, J. 2006. A new Big Five: Fundamental principles for an integrative science of personality. Am Psychol 61, 204-217.

McCrae, R. 2002. The maturation of personality psychology: Adult personality development and psychological well-being. J Res Pers 36, 307-317. 
McCrae, R., Costa, L. 1991. Adding liebe und arbeit: The full five-factor model and well-being. Pers Soc Psychol B 17, 227-232.

McCrae, R.R., Costa, P.T., Jr., Ostendorf, F., Angleitner, A., Hrebickova, M., Avia, M.D.,... Smith, P. B. 2000. Nature over nurture: Temperament, personality, and life span development. J Pers Soc Psychol 78, 173-186.

Macks, R., Reeve, R. 2007. The adjustment of non-disabled siblings of children with autism. J Autism Dev Disord 37, 1060-1067.

Mascha, K., Boucher, J. 2006. Preliminary investigation of a qualitative method of examining siblings' experiences of living with a child with ASD. Brit J Dev Disabil 52, 19-28.

Meyer, D. 1997. Views from Our Shoes: Growing up with a brother or sister with special needs. Woodbine House, Bethesda MD, USA.

Moore, M., Howard, V., McLaughlin, T. 2002. Siblings of children with disabilities: A review and analysis. Int J Spec Ed 17, 49-63.

Motamedi, S.H., Seyednour, R., Noorikhajavi, M., Afghah, S. 2007. A study in depression levels among mothers of disabled children. Iran Rehab J 5, 3-7.

Muris, P., Bos, A.E.R., Mayer, B., Verkade, R., Thewissen, V., Dell'Avvento, V. 2009. Relations among behavioral inhibition, Big Five personality factors, and anxiety disorder symptoms in non-clinical children. Pers Indiv Differ 46, 525-529.

Oruche, U., Gerkensmeyer, J., Stephan, L., Wheeler, C., Hanna, K. 2012. The described experience of primary caregivers of children with mental health needs. Arch Psychiat Nurs 26, 382-391. 
Pisula, E., Ziegart-Sadowska, K. 2015. Broader Autism Phenotype in siblings of children with ASD: A review. Int J Mol Sci 16, 13217-13258. DOI: 10.3390/ijms 160613217

Preacher, K., Hayes, A. 2008. Asymptotic and resampling strategies for assessing and comparing indirect effects in multiple mediator models. Behav Res Methods, 40, 879-891. DOI: 10.3758/BRM.40.3.879

Randall, P., Parker, J. 1999. Supporting the families of children with autism. John Wiley and Sons, New York, NY.

Redacted for blind review 2016a. Anxiety and depression symptomatology in adult siblings of individuals with different developmental disability diagnoses. Res Dev Disab 51, 116-125. DOI:10.1016/j.ridd.2015.12.017

Redacted for blind review 2016b. Perceived parenting styles fail to mediate between anxiety and attachment styles in adult siblings of individuals with developmental disabilities. J Autism Dev Disord 46, 3144-3154 DOI: 10.10007/s10803-016 2859-5

Powell, .T, Gallagher, P. 1993. Brothers and sisters: A special part of exceptional families. Paul H Brookes, Baltimore.

Preacher, K., Hayes, A. 2008. Asymptotic and resampling strategies for assessing and comparing indirect effects in multiple mediator models. Behav Res Methods, 40, 879-891. DOI: 10.3758/BRM.40.3.879

Ready, R., Robinson, M. 2008. Do older individuals adapt to their traits? Personality-emotion relations among younger and older adults. J Res Pers 42, 1020-1030. 
Rosenman, S., Rodgers, B. 2006. Childhood adversity and adult personality. Aust NZ J Psychiat 40, 482-490.

Rossiter, L., Sharpe, D. 2001. The siblings of individuals with mental retardation: A quantitative review of the literature. J Child Fam Stud 10, 65-84. http://dx.doi.org/10.1023/A: 1016629500708

Rothbart, M., Bates, J. 2006. Temperament, in: Damon, W., Lerner, R., Eisenberg, N. (Eds.), Handbook of child psychology: Vol. 3. Social, emotional, and personality development. Wiley, New York, pp. 99-166.

Rutter, M., Silberg, J. 2002. Gene-environment interplay in relation to emotional and behavioral disturbance. Ann Rev Psychol 53, 463-490. DOI: 10.1146/annurev.psych.53.100901.135223

Scott, J., Williams, J., Brittlebank, A., Ferrier, I. 1995. The relationship between premorbid neuroticism, cognitive dysfunction and persistence of depression: A 1 year follow-up. J Affect Disorders 33, 167-172.

Seltzer, M., Orsmond, G., Esbensen, A. 2009. Siblings of individuals with an autism spectrum disorder: Sibling relationships and wellbeing in adolescence and adulthood. Autism 13, 59-80.

Shiner, R., Caspi, A. 2003. Personality differences in childhood and adolescence: Measurement, development, and consequence. J Child Psychol Psychiat 44, 2-32.

Slobodskaya, H. R., Gartstein, M. A., Nakagawa, A., Putnam, S. P. 2012. Early temperament in Japan, the United States and Russia. J Cross Cult Psychol 44, 438-460. DOI: 10.1177/0022022112453316 
Snaith, R. 2003. The Hospital Anxiety and Depression Scale. Health and Quality of Life Outcomes, 1, 29.

Spinhoven, P., Roelofs, K., Hovens, J., Elzinga, B., van Oppen, P., Zitman, F., Pennix, B. 2011. Personality, life events and the course of anxiety and depression. Europ J Pers 25, 445-453.

Srivastava, S., John, O., Gosling, S., Potter, J. 2003. Development of personality in early and middle adulthood: Set like plaster or persistent change? J Pers Soc Psychol 84, 1041-1053.

Trull, T., McCrae, R. 2005. A five-factor perspective on personality disorder research, in: Costa, P. Jr., Widiger, T. (Eds.), Personality disorders and the five factor model of personality. Washington, DC, USA, American Psychological Association, pp. 45-57.

Wakabayashi, A., Baron-Cohen. S., Wheelwright, S. 2006. Are autistic traits an independent personality dimension? A study of the Autism-Spectrum Quotient (AQ) and the NEO-PI-R. Pers Indiv Differ 41, 873-883. https://doi.org/10.1016/j.paid.2006.04.003

Watson, D., Gamez, W., Simms, L. 2005. Basic dimensions of temperament and their relation to anxiety and depression: A symptom based perspective. J Res Pers 39, 46-66.

Widiger, T., Anderson, K. 2003. Personality and depression in women. J Affect Disorders 74, 5966. 
Wilson, C., McGillivray, J., Zetlin, A. 1992. The relationship between attitude to disabled sibling and rating of behavioral competency. J Intell Disabil Res 36, 325 336.

Wolfe, B., Song, J., Greenberg, J., Mailick, M. 2014. Ripple effects of developmental disabilities and mental illness on nondisabled adult siblings. Soc Sci Med 108, 1 9. http://dx.doi.org/10.1016/j.socscimed.2014.01.021

Ypofanti, M., Zisi, V., Zourbanos, N., Mouchtouri, B., Tzanne, P., Theodorakis, Y., Lyrakos, G. 2015. Psychometric properties of the International Personality Item Pool Big-Five Personality Questionnaire for the Greek population. Health Psychol Res 3, 2206. doi: 10.4081/hpr.2015.2206

Zheng, L., Goldberg, L., Zheng, Y., Zhao, Y., Tang, Y., Liu, L. 2008. Reliability and concurrent validation of the IPIP Big-Five Factor Markers in China: Consistencies in factor structure between internet-obtained heterosexual and homosexual samples. Pers Indiv Dif 45, 649-654.

Zigmond, A., Snaith, R. 1983. The Hospital Anxiety and Depression Scale. Psychiat Scand 67, 361-437. 
